\title{
Bilinearization and new soliton solutions of Whitham-Broer-Kaup equations with time-dependent coefficients
}

\author{
Sheng Zhang*, Zhaoyu Wang \\ School of Mathematics and Physics, Bohai University, Jinzhou 121013, China.
}

Communicated by D. Baleanu

\begin{abstract}
In this paper, Whitham-Broer-Kaup (WBK) equations with time-dependent coefficients are exactly solved through Hirota's bilinear method. To be specific, the WBK equations are first reduced into a system of variable-coefficient Ablowitz-KaupNewell-Segur (AKNS) equations. With the help of the AKNS equations, bilinear forms of the WBK equations are then given. Based on a special case of the bilinear forms, new one-soliton solutions, two-soliton solutions, three-soliton solutions and the uniform formulae of $n$-soliton solutions are finally obtained. It is graphically shown that the dynamical evolutions of the obtained one-, two- and three-soliton solutions possess time-varying amplitudes in the process of propagations. (C)2017 All rights reserved.
\end{abstract}

Keywords: Bilinear form, soliton solution, WKB equations with time-dependent coefficients, Hirota's bilinear method. 2010 MSC: 35Q51, 35Q53, 35Q99.

\section{Introduction}

In nonlinear science, many physical phenomena such as fluid dynamics, plasma physics and nonlinear optics are often related to nonlinear partial differential equations (PDEs). Researchers often investigate solutions of such nonlinear PDEs (see for examples [17-21]) to gain more insight into these physical phenomena for further applications. Since the celebrated Korteweg-de Vries (KdV) equation was exactly solved by Gardner et al. [13], finding exact solutions of nonlinear PDEs has gradually developed into one of the most important and significant directions and many effective methods have been proposed such as the inverse scattering method [1, 61, 66, 69], Hirota's bilinear method [15], Bäcklund transformation [33], Darboux transformation [31, 47, 64], Painlevé expansion [46, 59, 60], homogeneous balance method [44], subsidiary equation method [12, 22, 67, 68], first integral method [4], residual power series method [23], and the exp-function method $[14,55,56]$.

As a direct method, Hirota's bilinear method [15] proposed in 1971 has been widely used to construct multi-soliton solutions of many nonlinear PDEs like those in [5, 16, 29, 32, 45, 62, 63, 65]. Besides, Hirota's bilinear method [15] and Darboux transformation [31] are two of the most powerful techniques for constructing rogue-wave solutions $[6,28,50]$ of nonlinear PDEs. The key step of Hirota's bilinear method is to convert the given nonlinear PDE into the so-called bilinear form. For such bilinear forms, there is

\footnotetext{
*Corresponding author

Email addresses: szhangchina@126.com (Sheng Zhang), 1174833500@qq.com (Zhaoyu Wang)
} 
no general rule to follow and one often tries to take some transformations like rational transformation or logarithmic transformation. Recently, Hirota's bilinear method was extended in a uniform way to all the nonlinear PDEs contained in the isospectral AKNS hierarchy [10], the variable-coefficient KdV hierarchy [58] and the modified $\mathrm{KdV}(\mathrm{mKdV})$ hierarchy [72]. When the inhomogeneities of media and nonuniformities of boundaries are taken into account, the variable-coefficient PDEs could describe more realistic physical phenomena than their constant-coefficient counterparts. Therefore, how to generalize the existing methods to construct exact solutions especially soliton solutions [42, 43] of nonlinear PDEs with variable coefficients is worthy of exploring. In the present paper, we shall extend Hirota's bilinear method to construct new multi-soliton solutions of the following new WBK equations with time-dependent coefficients [30]:

$$
\begin{gathered}
\mathfrak{u}_{\mathrm{t}}+\gamma_{1}(\mathrm{t}) \mathrm{u} u_{x}+\gamma_{2}(\mathrm{t}) v_{x}+\gamma_{3}(\mathrm{t}) \mathrm{u}_{x x}=0, \\
v_{\mathrm{t}}+\gamma_{4}(\mathrm{t}) \mathrm{u}_{x} v+\gamma_{4}(\mathrm{t}) \mathrm{u} v_{x}-\gamma_{5}(\mathrm{t}) v_{x x}+\gamma_{6}(\mathrm{t}) \mathrm{u}_{x x x}=0,
\end{gathered}
$$

where $\gamma_{i}(t)(i=1,2, \cdots, 6)$ are arbitrary smooth functions of $t$, which represent different dispersion and dissipation forces. Given different $\gamma_{i}(t)(i=1,2, \cdots, 6)$, equations (1.1) and (1.2) convert into some well-known equations. If $\gamma_{i}(t)=h_{i}(i=1,2, \cdots, 6)$ are all constants, then (1.1) and (1.2) become the generalized WBK equations [30]:

$$
\begin{gathered}
u_{t}+h_{1} u u_{x}+h_{2} v_{x}+h_{3} u_{x x}=0, \\
v_{t}+h_{4} u_{x} v+h_{4} u v_{x}-h_{5} v_{x x}+h_{6} u_{x x x}=0 .
\end{gathered}
$$

When $\gamma_{1}(t)=\gamma_{2}(t)=\gamma_{4}(t)=-1, \gamma_{3}(t)=\gamma_{5}(t)=1 / 2$ and $\gamma_{6}(t)=0$, equations (1.1) and (1.2) give the approximate equations for long water waves [51]:

$$
\begin{gathered}
u_{t}-u u_{x}-v_{x}+\frac{1}{2} u_{x x}=0, \\
v_{t}-(u v)_{x}-\frac{1}{2} v_{x x}=0 .
\end{gathered}
$$

When $\gamma_{1}(t)=\gamma_{2}(t)=\gamma_{4}(t)=1, \gamma_{3}(t)=\gamma_{5}(t)=\beta$ and $\gamma_{6}(t)=\alpha$ are all constants, equations (1.1) and (1.2) transform into the WBK equations in shallow water [52]:

$$
\begin{gathered}
u_{t}+u u_{x}+v_{x}+\beta u_{x x}=0, \\
v_{t}+(u v)_{x}+\alpha u_{x x x}-\beta v_{x x}=0 .
\end{gathered}
$$

When $\gamma_{1}(t)=\gamma_{4}(t)=2, \gamma_{2}(t)=\gamma_{6}(t)=-1 / 2$ and $\gamma_{3}(t)=\gamma_{5}(t)=0$, equations (1.1) and (1.2) degenerate into the Boussinesq-Burgers (BB) equations [25]:

$$
\begin{gathered}
u_{t}+2 u u_{x}-\frac{1}{2} v_{x}=0, \\
v_{t}-\frac{1}{2} u_{x x x}+2(u v)_{x}=0 .
\end{gathered}
$$

When $\gamma_{1}(t)=\gamma_{2}(t)=1, \gamma_{3}(t)=\gamma_{5}(t)=0$ and $\gamma_{6}(t)=1$, equations (1.1) and (1.2) change into the variant Boussinesq equations [51]:

$$
\begin{gathered}
u_{t}+u u_{x}+v_{x}=0, \\
v_{t}+(u v)_{x}+u_{x x x}=0 .
\end{gathered}
$$

When $\gamma_{1}(t)=\gamma_{2}(t)=\gamma_{4}(t)=2 \alpha(t), \gamma_{3}(t)=\gamma_{5}(t)=-\alpha(t), \gamma_{6}(t)=0$, equations (1.1) and (1.2) turn into the variable-coefficient Broer-Kaup (BK) equations [53]:

$$
\begin{gathered}
u_{x t}-\alpha(t)\left[u_{x x x}-2\left(u u_{x}\right)_{x}-2 v_{x x}\right]=0, \\
v_{t}+\alpha(t)\left[v_{x x}+2(u v)_{x}\right]=0 .
\end{gathered}
$$

In 2014, Liu and Liu [30] obtained some symmetries and similarity reductions of (1.3) and (1.4) by apply- 
ing direct symmetry method. Based on the obtained symmetries, Liu and Liu [30] obtained some new solutions including rational solutions, hyperbolic function solutions, trigonometric function solutions and Jacobi elliptic function solutions of (1.1) and (1.2). As far as we know, there are no multi-soliton solutions and other solutions of (1.1), (1.2), (1.3), (1.4) have been reported. By using the extend homogeneous balance method, Yan and Liu [51] obtained trigonometric function solutions, $n$-resonance plane solitary wave solutions and non-traveling wave solutions of (1.5) and (1.6) and (1.11) and (1.12).

In recent years, most researchers focus on the constant-coefficient WBK (1.7) and (1.8). More specifically, Yan and Zhang [52] obtained solitary wave solutions, periodic wave solutions and the combined formal solitary wave solutions by introducing Riccati equation and a new generalized transformation. Chen and Wang [7] obtained many trigonometric periodic and hyperbolic function solutions, trigonometric periodic and rational function solutions, hyperbolic and rational function solutions by proposing multiple Riccati equations rational expansion method. Zhang [57] obtained solitary wave solutions, periodic wave solutions and combined formal solitary wave solutions by using an improved Riccati equations method. Chen et al. [9] obtained a series of exact solutions which include rational form solitary wave solution, rational form triangular periodic wave solutions and rational wave solutions as special cases by presenting elliptic equation rational expansion method. Mohebbi et al. [34] obtained numerical solutions by proposing numerical schemes which are based on the fourth-order time-stepping schemes in combination with discrete Fourier transform. Shen et al. [40] obtained solitary wave solutions, kink wave solutions and periodic wave solutions by using bifurcation method. El-Sayed and Kaya [11] obtained explicit and numerical traveling wave solutions containing blow-up solutions and periodic solutions by using a decomposition method. Chen et al. [8] obtained more types of traveling wave solutions including polynomial, exponential, solitary wave, rational, triangular periodic, Jacobi and Weierstrass doubly periodic solutions by proposing a generalized method. $\mathrm{Xu}$ and $\mathrm{Li}$ [49] obtained some singular traveling wave solutions by improving an auxiliary equation method. Jiao and Zhang [24] obtained exact traveling wave solutions by proposing an extended method with a more general transformation. Xie et al. [48] obtained four pairs of solutions which contain blow-up solutions and periodic solutions by using hyperbolic function method and $\mathrm{Wu}$ elimination method. Mohyud-Din et al. [35] obtained explicit and numerical traveling wave solutions which contain blow-up solutions and periodic solutions by using homotopy perturbation method. Rafei and Daniali [36] obtained explicit traveling wave solutions including blow-up and periodic solutions by using variational iteration method. Song et al. [41] obtained kink-shaped solutions, blow-up solutions, periodic blow-up solutions and solitary wave solutions by using bifurcation method and qualitative theory of dynamic systems. Arshad et al. [2] obtained traveling wave solutions in the form of solitons, bell and anti-bell periodic, bright and dark solitary wave by applying a modified extended direct algebraic method. Lin et al. [27] obtained multi-soliton solutions by means of Wronskian technique and symbolic computation.

With the help of Riccati equation and its some special solutions, Khalfallah [25] obtained hyperbolic function solutions and rational solutions of (1.9) and (1.10). By using the compatibility method, Yan and Zhou [53] obtained many explicit solutions of the Boussinesq-Burgers (1.13) and (1.14), which include solutions expressed by error function, Bessel function, exponential function and Airy function. As a special case of Zhang and Zhang's work in [70], $n$-soliton solutions of the variable-coefficient BK (1.13) and (1.14) can be reached.

The rest of the paper is organized as follows. In Section 2, we first take appropriate transformations to reduce (1.1) and (1.2) into the variable-coefficient AKNS equations. Then the variable-coefficient AKNS equations are bilinearized so that we arrive at the bilinear forms of (1.1) and (1.2). In Section 3, starting from a special case of the obtained bilinear forms, we construct one-soliton, two-soliton, and three-soliton solutions of (1.1) and (1.2). Based on the obtained soliton solutions, we then summarize a uniform formula for the explicit $n$-soliton solutions of (1.1) and (1.2). In addition, some spatial structures and propagations of the obtained one-, two- and three-soliton solutions are shown by figures. In Section 4, we conclude this paper. 


\section{Bilinearization}

For (1.1) and (1.2), we have the following Theorem 2.1.

Theorem 2.1. Suppose that

$$
\begin{gathered}
u=a_{0} \frac{A_{x}}{A}, \\
v=-a_{0}^{2} \frac{\gamma_{1}(t)}{\gamma_{2}(t)} A B+a_{0} \frac{a_{0} \gamma_{1}(t)-2 \gamma_{3}(t)}{2 \gamma_{2}(t)}\left(-\frac{A_{x}^{2}}{A^{2}}+\frac{A_{x x}}{A}\right),
\end{gathered}
$$

where $\mathrm{a}_{0}$ is an arbitrary constant, $\mathrm{A}$ and $\mathrm{B}$ are smooth functions of $\mathrm{x}$ and $\mathrm{t}$, the time-dependent-coefficient WBK (1.1) and (1.2) reduce then into the variable-coefficient AKNS equations:

under the constraints

$$
\begin{aligned}
& A_{t}=\frac{1}{2} a_{0} \gamma_{1}(t)\left(2 A^{2} B-A_{x x}\right), \\
& B_{t}=\frac{1}{2} a_{0} \gamma_{1}(t)\left(-2 A B^{2}+B_{x x}\right),
\end{aligned}
$$

$$
\begin{gathered}
\gamma_{4}(t)=\gamma_{1}(t), \quad \gamma_{5}(t)=\gamma_{3}(t), \quad \gamma_{6}(t)=\frac{a_{0}^{2} \gamma_{1}^{2}(t)-4 \gamma_{3}^{2}(t)}{4 \gamma_{2}(t)}, \\
\gamma_{3}^{\prime}(t)=\frac{\gamma_{1}^{\prime}(t) \gamma_{3}(t)}{\gamma_{1}(t)}, \quad \gamma_{2}^{\prime}(t)=\frac{\gamma_{1}^{\prime}(t) \gamma_{2}(t)}{\gamma_{1}(t)} .
\end{gathered}
$$

Proof. We take the following transformations

$$
\begin{gathered}
u=a(t)(\ln A)_{x} \\
v=b(t)(\ln A)_{x x}+c(t) A B,
\end{gathered}
$$

and substitute (2.7) and (2.8) into (1.1) and (1.2), here $a(t), b(t)$ and $c(t)$ are functions of $t$ to be determined. A direct computation tells that if

$$
a(t)=a_{0}, \quad b(t)=a_{0} \frac{a_{0} \gamma_{1}(t)-2 \gamma_{3}(t)}{2 \gamma_{2}(t)}, \quad c(t)=-a_{0}^{2} \frac{\gamma_{1}(t)}{\gamma_{2}(t)},
$$

then (1.1) and (1.2) reduce into (2.3) and (2.4) under the constrains (2.5) and (2.6). Thus, the proof is end.

For the bilinear forms of (1.1) and (1.2), we have the following Theorem 2.2.

Theorem 2.2. Let (2.5) and (2.6) hold, the time-dependent-coefficient WBK (1.1) and (1.2) possess the bilinear forms

$$
\begin{aligned}
& D_{t} g \cdot f=\frac{1}{2} a_{0} \gamma_{1}(t)\left[-D_{x}^{2} g \cdot f+\frac{g}{f}\left(D_{x}^{2} f \cdot f+2 g h\right)\right], \\
& D_{t} h \cdot f=\frac{1}{2} a_{0} \gamma_{1}(t)\left[D_{x}^{2} h \cdot f-\frac{h}{f}\left(D_{x}^{2} f \cdot f+2 g h\right)\right],
\end{aligned}
$$

where $f=f(x, t), g=g(x, t), h=h(x, t), D_{x}$ and $D_{t}$ are Hirota's differential operators defined by

$$
D_{x}^{m} D_{t}^{n} F(x, t) \cdot G(x, t)=\left.\left(\partial_{x}-\partial_{x^{\prime}}\right)^{m}\left(\partial_{t}-\partial_{t^{\prime}}\right)^{n} F(x, t) \cdot G\left(x^{\prime}, t^{\prime}\right)\right|_{x^{\prime}=x, t^{\prime}=t} .
$$

Proof. In view of (2.3) and (2.4), we suppose that

$$
A=\frac{g}{f}, \quad B=\frac{h}{f}
$$

Equations (2.3) and (2.4) are then converted into

$$
\begin{aligned}
& f g_{t}-f_{t} g=\frac{1}{2} a_{0} \gamma_{1}(t)\left(-g_{x x} f+2 g_{x} f_{x}+f_{x x} g-\frac{2 f_{x}^{2} g}{f}+\frac{2 g^{2} h}{f}\right), \\
& f h_{t}-f_{t} h=\frac{1}{2} a_{0} \gamma_{1}(t)\left(f h_{x x}-2 f_{x} h_{x}-f_{x x} h+\frac{2 f_{\chi}^{2} h}{f}-\frac{2 g h^{2}}{f}\right),
\end{aligned}
$$

the bilinear forms of which are namely (2.9) and (2.10). It is easy to see from (2.1), (2.2) and (2.11) that if 
we take the transformations

$$
\begin{gathered}
u=a_{0} \frac{g_{x} f-f_{x} g}{f g}, \\
v=-a_{0}^{2} \frac{\gamma_{1}(t)}{\gamma_{2}(t)} \frac{g h}{f^{2}}+a_{0} \frac{a_{0} \gamma_{1}(t)-2 \gamma_{3}(t)}{2 \gamma_{2}(t)}\left(\frac{f_{x}^{2}}{f^{2}}-\frac{g_{x}^{2}}{g^{2}}-\frac{f_{x x}}{f}+\frac{g_{x x}}{g}\right),
\end{gathered}
$$

then (1.1) and (1.2) are converted into the bilinear forms (2.9) and (2.10). We complete the proof.

\section{Multi-soliton solutions}

To construct multi-soliton solutions of (1.1) and (1.2) conveniently, we set

$$
D_{x}^{2} f \cdot f+2 g h=0
$$

the bilinear forms (2.9) and (2.10) are then reduced into

$$
\begin{aligned}
& D_{t} g \cdot f=-\frac{1}{2} a_{0} \gamma_{1}(t) D_{x}^{2} g \cdot f, \\
& D_{t} h \cdot f=\frac{1}{2} a_{0} \gamma_{1}(t) D_{x}^{2} h \cdot f .
\end{aligned}
$$

Based on the bilinear forms (3.2) and (3.3) under the condition (3.1), in what follows we construct multi-soliton solutions of (1.1) and (1.2). For this purpose, we suppose that

$$
\begin{aligned}
& f=1+\varepsilon^{2} f^{(2)}+\varepsilon^{4} f^{(4)}+\ldots+\varepsilon^{2 j} f^{(2 j)}+\cdots, \\
& g=\varepsilon g^{(1)}+\varepsilon^{3} g^{(3)}+\ldots+\varepsilon^{2 j+1} g^{(2 j+1)}+\cdots, \\
& h=\varepsilon h^{(1)}+\varepsilon^{3} h^{(3)}+\ldots+\varepsilon^{2 j+1} h^{(2 j+1)}+\cdots
\end{aligned}
$$
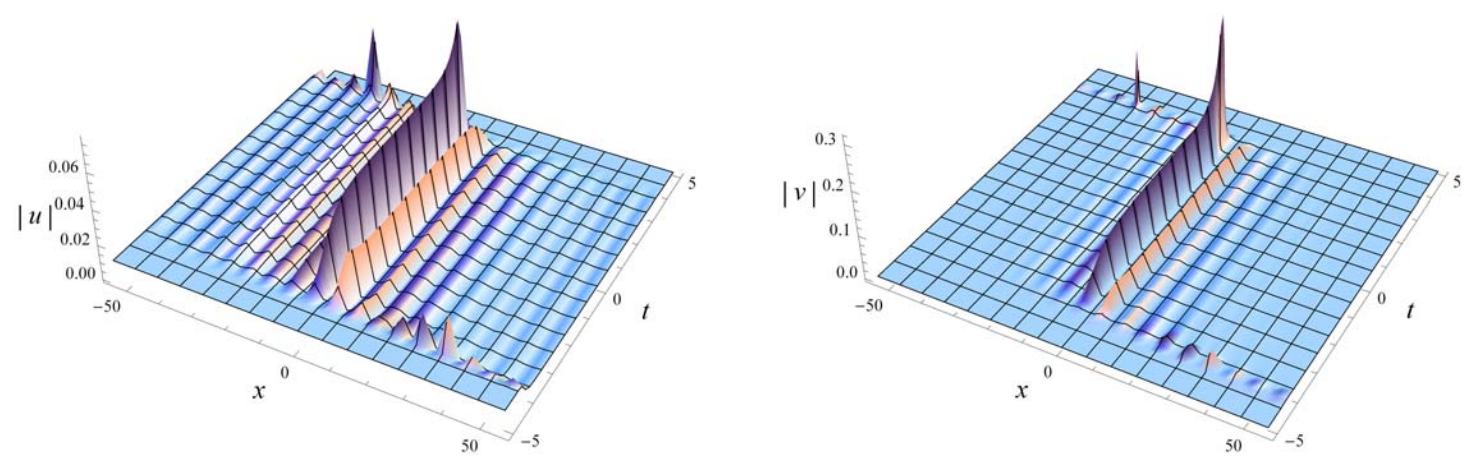

Figure 1: Spatial structures of one-soliton solutions (3.18) and (3.19).

Substituting (3.4), (3.5), (3.6) into (3.1), (3.2), (3.3) and then collecting the coefficients of the same order of $\varepsilon$ yields a system of differential equations (SDEs)

$$
g_{t}^{(1)}+\frac{1}{2} a_{0} \gamma_{1}(t) g_{x x}^{(1)}=0,
$$




$$
\begin{gathered}
h_{t}^{(1)}-\frac{1}{2} a_{0} \gamma_{1}(t) h_{x x}^{(1)}=0, \\
f_{x x}^{(2)}+g^{(1)} h^{(1)}=0 \\
g_{t}^{(3)}+\frac{1}{2} a_{0} \gamma_{1}(t) g_{x x}^{(3)}=-\left[D_{t}+\frac{1}{2} a_{0} \gamma_{1}(t) D_{x}^{2}\right] g^{(1)} \cdot f^{(2)}, \\
h_{t}^{(3)}-\frac{1}{2} a_{0} \gamma_{1}(t) h_{x x}^{(3)}=-\left[D_{t}-\frac{1}{2} a_{0} \gamma_{1}(t) D_{x}^{2}\right] h^{(1)} \cdot f^{(2)}, \\
2 f_{x x}^{(4)}=-D_{x}^{2} f^{(2)} \cdot f^{(2)}-2\left(g^{(1)} h^{(3)}+g^{(3)} h^{(1)}\right), \\
g_{t}^{(5)}+\frac{1}{2} a_{0} \gamma_{1}(t) g_{x x}^{(5)}=-\left[D_{t}+\frac{1}{2} a_{0} \gamma_{1}(t) D_{x}^{2}\right]\left(g^{(1)} \cdot f^{(4)}+g^{(3)} f^{(2)}\right), \\
h_{t}^{(5)}-\frac{1}{2} a_{0} \gamma_{1}(t) h_{x x}^{(5)}=-\left[D_{t}-\frac{1}{2} a_{0} \gamma_{1}(t) D_{x}^{2}\right]\left(h^{(1)} \cdot f^{(4)}+h^{(3)} f^{(2)}\right), \\
f_{x x}^{(6)}=-D_{x}^{2} f^{(2)} \cdot f^{(4)}-\left(g^{(1)} h^{(5)}+g^{(3)} h^{(3)}+g^{(5)} h^{(1)}\right),
\end{gathered}
$$

and so forth.

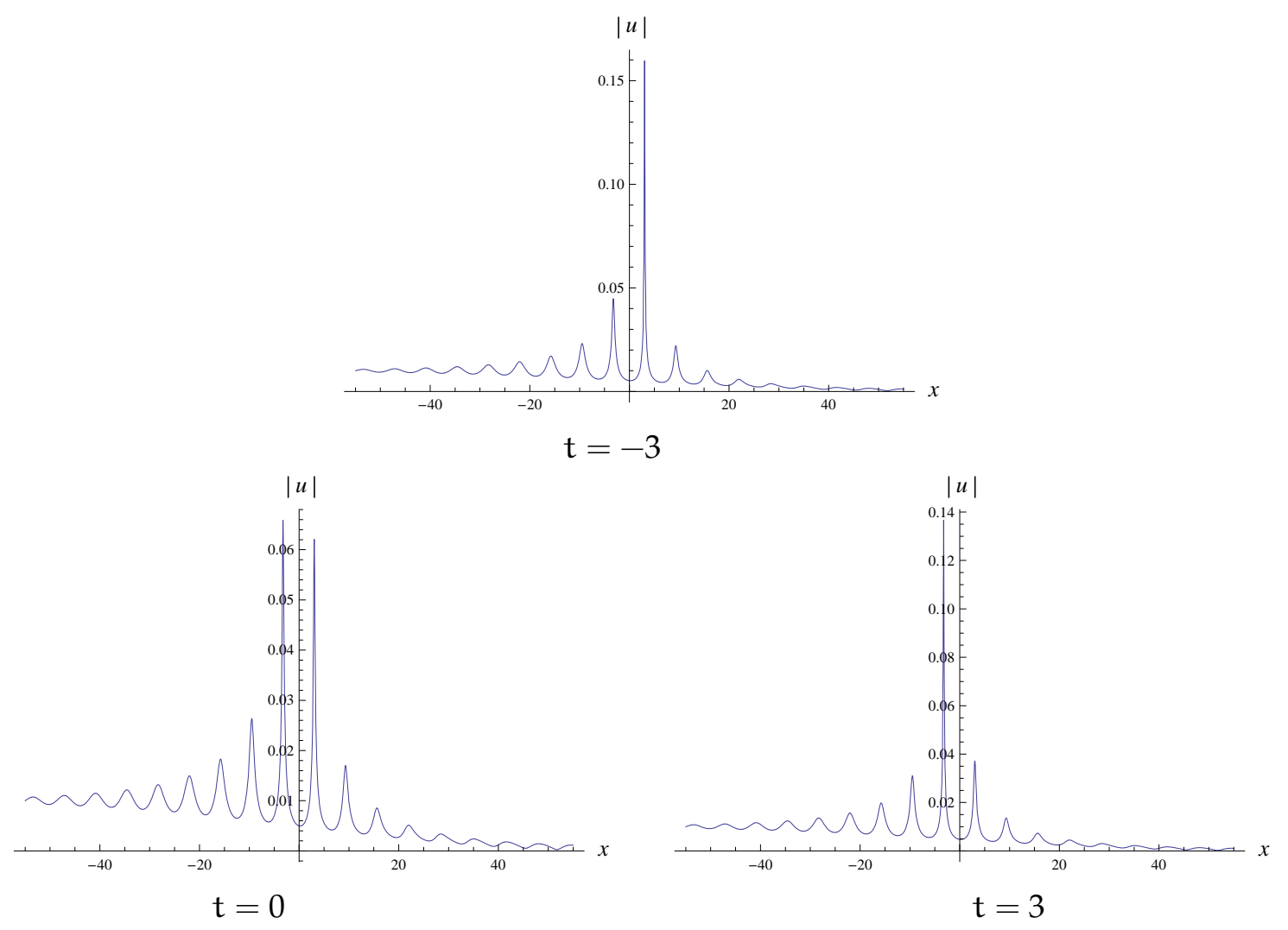

Figure 2: Dynamical evolutions of one-soliton solution (3.18).

If let

$$
\begin{aligned}
& g^{(1)}=e^{\xi_{1}}, \quad \xi_{1}=k_{1} x-\frac{1}{2} a_{0} k_{1}^{2} \int \gamma_{1}(t) d t, \\
& h^{(1)}=e^{\eta_{1}}, \quad \eta_{1}=l_{1} x+\frac{1}{2} a_{0} l_{1}^{2} \int \gamma_{1}(t) d t
\end{aligned}
$$


be two solutions of (3.7) and (3.8), then from (3.9) we have

$$
f^{(2)}=e^{\xi_{1}+\eta_{1}+\theta_{13}}, \quad e^{\theta_{13}}=-\frac{1}{\left(k_{1}+l_{1}\right)^{2}} .
$$
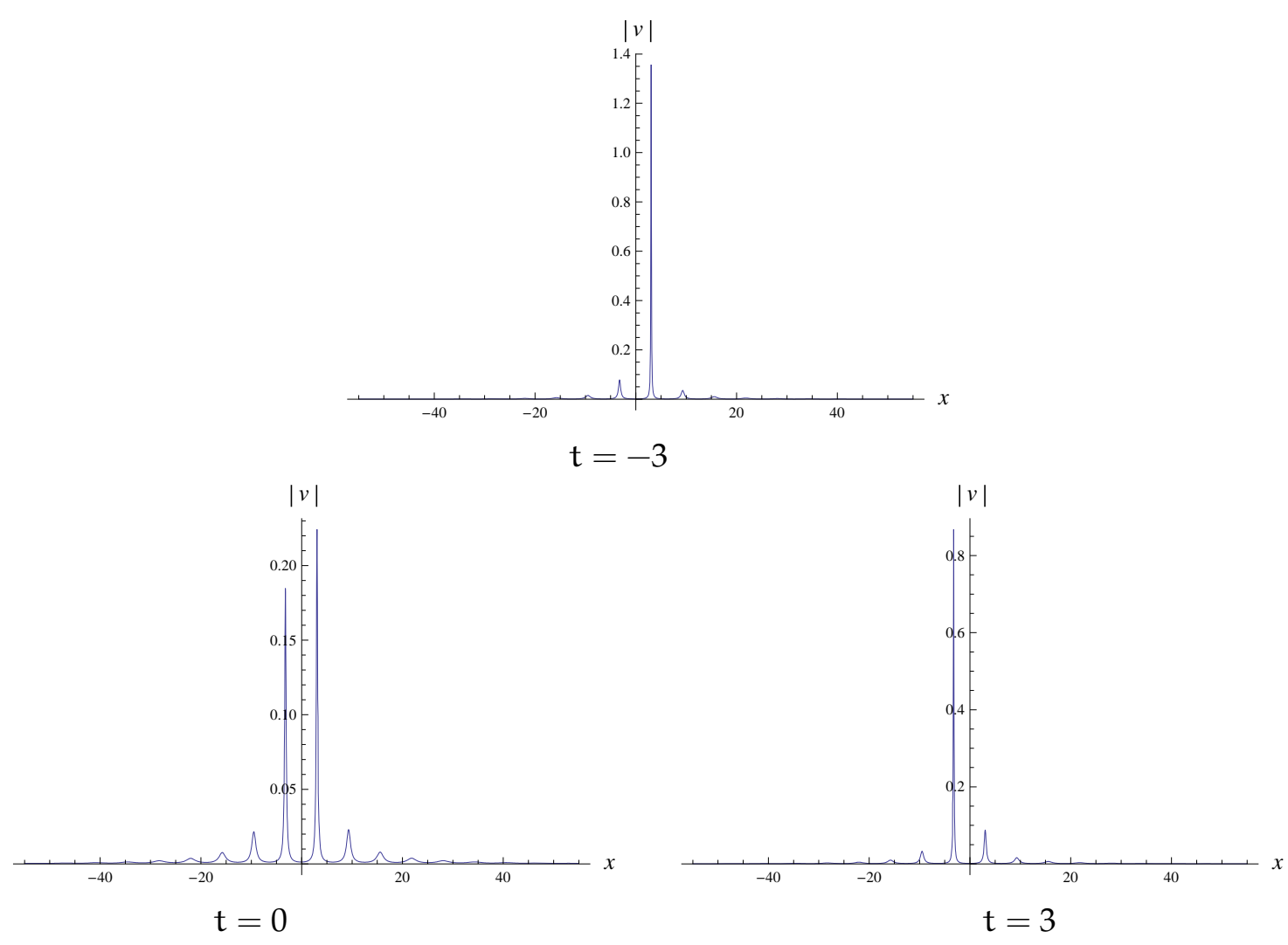

Figure 3: Dynamical evolutions of one-soliton solutions (3.19).

It is easy to see that if $g^{(3)}=h^{(3)}=f^{(4)}=\cdots=0$, then (3.10), (3.11), (3.12), (3.13), (3.14), (3.15) and those unwritten ones in above SDEs all hold. In this case, (3.4), (3.5), (3.6) are truncated into finite terms. Further selecting $\varepsilon=1$ and writing

$$
f_{1}=1+e^{\xi_{1}+\eta_{1}+\theta_{13}}, \quad g_{1}=e^{\xi_{1}}, \quad h_{1}=e^{\eta_{1}},
$$

we then obtain one-soliton solutions of (1.1) and (1.2):

$$
\begin{gathered}
u=a_{0} \frac{k_{1}-l_{1} e^{\xi_{1}+\eta_{1}+\theta_{13}}}{1+e^{\xi_{1}+\eta_{1}+\theta_{13}}}, \\
v=-a_{0}\left[2 a_{0}-a_{0} \gamma_{1}(t)+2 \gamma_{3}(t)\right] \frac{e^{\xi_{1}+\eta_{1}}}{2 \gamma_{2}(t)\left(1+e^{\xi_{1}+\eta_{1}+\theta_{13}}\right)^{2}} .
\end{gathered}
$$

In Figure 1, the spatial structures of one-soliton solutions (3.18) and (3.19) are shown, where the parameters are selected as $k_{1}=i, l_{1}=0.05, a_{0}=0.01, \gamma_{1}(t)=0.5 e^{0.5 t^{2}}, \gamma_{2}(t)=2 e^{0.5 t^{2}}, \gamma_{3}(t)=e^{0.5 t^{2}}$. We use Figures 2 and 3 to describe the corresponding dynamical evolutions of one-soliton solutions (3.18) and (3.19) at times $t=-3, t=0$ and $t=3$. It can be seen from Figures $1-3$ that one-soliton solutions (3.18) and (3.19) possess time-varying amplitudes in the process of propagations.

We next construct two-soliton solutions of (1.1) and (1.2). Selecting

$$
g^{(1)}=e^{\xi_{1}}+e^{\xi_{2}}, \quad \xi_{i}=k_{i} x-\frac{1}{2} a_{0} k_{i}^{2} \int \gamma_{1}(t) d t, \quad(i=1,2),
$$




$$
h^{(1)}=e^{\eta_{1}}+e^{\eta_{2}}, \quad \eta_{i}=l_{i} x+\frac{1}{2} a_{0} l_{i}^{2} \int \gamma_{1}(t) d t, \quad(i=1,2),
$$

which satisfy (3.7) and (3.8), from (3.9), (3.10), (3.11), (3.12) we then have

$$
\begin{aligned}
& f^{(2)}=e^{\xi_{1}+\eta_{1}+\theta_{13}}+e^{\xi_{1}+\eta_{2}+\theta_{14}}+e^{\xi_{2}+\eta_{1}+\theta_{23}}+e^{\xi_{2}+\eta_{2}+\theta_{24},}, \\
& g^{(3)}=e^{\xi_{1}+\xi_{2}+\eta_{1}+\theta_{12}+\theta_{13}+\theta_{23}}+e^{\xi_{1}+\xi_{2}+\eta_{2}+\theta_{12}+\theta_{14}+\theta_{24},}, \\
& h^{(3)}=e^{\xi_{1}+\eta_{1}+\eta_{2}+\theta_{13}+\theta_{14}+\theta_{34}}+e^{\xi_{2}+\eta_{1}+\eta_{2}+\theta_{23}+\theta_{24}+\theta_{34},}, \\
& f^{(4)}=e^{\xi_{1}+\xi_{2}+\eta_{1}+\eta_{2}+\theta_{13}+\theta_{14}+\theta_{34}+\theta_{23}+\theta_{24}+\theta_{34},}
\end{aligned}
$$

where

$$
e^{\theta_{12}}=-\left(k_{1}-k_{2}\right)^{2}, \quad e^{\theta_{34}}=-\left(l_{1}-l_{2}\right)^{2}, \quad e^{\theta_{i(j+2)}}=-\frac{1}{\left(k_{i}+l_{j}\right)^{2}}, \quad(i, j=1,2) .
$$
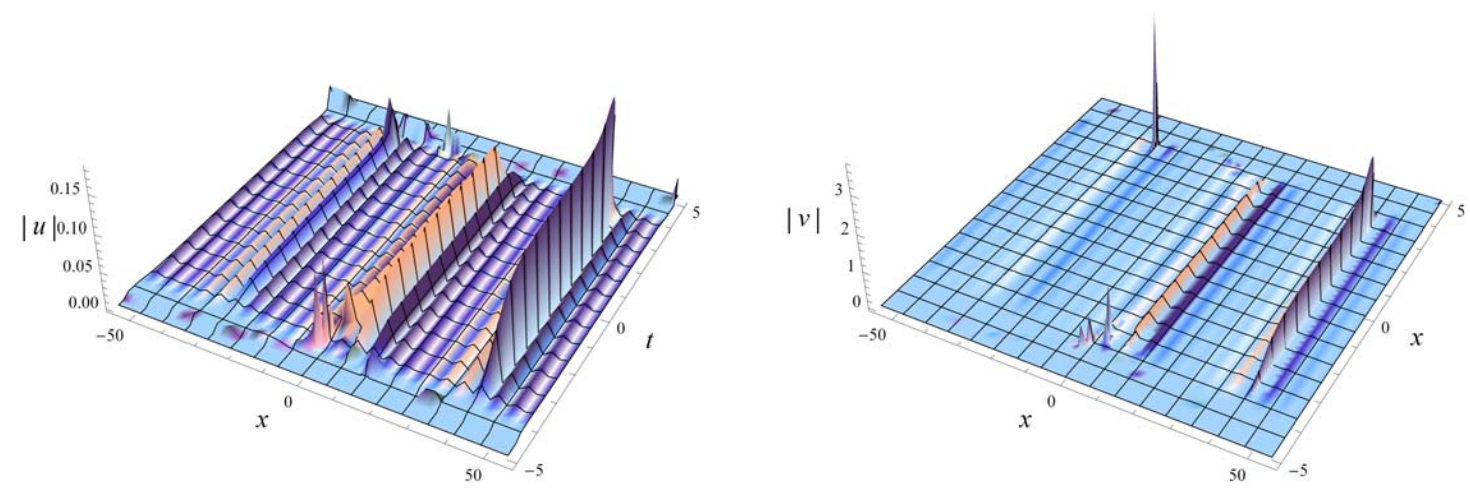

Figure 4: Spatial structures of two-soliton solutions (3.20) and (3.21).

If $g^{(5)}=h^{(5)}=f^{(6)}=\cdots=0$, then we can see that (3.13), (3.14), (3.15) and those unwritten ones in above SDEs all hold. In this case, we truncate (3.4), (3.5), (3.6) into finite terms. We further select $\varepsilon=1$ and write

$$
\begin{aligned}
g_{2}= & e^{\xi_{1}}+e^{\xi_{2}}+e^{\xi_{1}+\xi_{2}+\eta_{1}+\theta_{12}+\theta_{13}+\theta_{23}}+e^{\xi_{1}+\xi_{2}+\eta_{2}+\theta_{12}+\theta_{14}+\theta_{24}}, \\
h_{2}= & e^{\eta_{1}}+e^{\eta_{2}}+e^{\xi_{1}+\eta_{1}+\eta_{2}+\theta_{13}+\theta_{14}+\theta_{34}}+e^{\xi_{2}+\eta_{1}+\eta_{2}+\theta_{23}+\theta_{24}+\theta_{34}}, \\
f_{2}= & 1+e^{\xi_{1}+\eta_{1}+\theta_{13}}+e^{\xi_{1}+\eta_{2}+\theta_{14}}+e^{\xi_{2}+\eta_{1}+\theta_{23}}+e^{\xi_{2}+\eta_{2}+\theta_{24}} \\
& +e^{\xi_{1}+\xi_{2}+\eta_{1}+\eta_{2}+\theta_{13}+\theta_{14}+\theta_{34}+\theta_{23}+\theta_{24}+\theta_{34}},
\end{aligned}
$$

and hence obtain two-soliton solutions of (1.1) and (1.2):

$$
\begin{gathered}
u=a_{0} \frac{g_{2 x} f_{2}-f_{2 x} g_{2}}{f_{2} g_{2}}, \\
v=-a_{0}^{2} \frac{\gamma_{1}(t)}{\gamma_{2}(t)} \frac{g_{2} h_{2}}{f_{2}^{2}}+a_{0} \frac{a_{0} \gamma_{1}(t)-2 \gamma_{3}(t)}{2 \gamma_{2}(t)}\left(\frac{f_{2 x}^{2}}{f_{2}^{2}}-\frac{g_{2 x}^{2}}{g_{2}^{2}}-\frac{f_{2 x x}}{f_{2}}+\frac{g_{2 x x}}{g_{2}}\right) .
\end{gathered}
$$

The spatial structures of two-soliton solutions (3.20) and (3.21) are shown in Figure 4 by selecting the parameters as $k_{1}=i, k_{2}=0.3 i, l_{1}=4, l_{2}=-1.2, a_{0}=0.008, \gamma_{1}(t)=0.5 e^{0.5 t^{2}}, \gamma_{2}(t)=2 e^{0.5 t^{2}}$, $\gamma_{3}(t)=e^{0.5 t^{2}}$. Figures 5 and 6 are used to describe the corresponding dynamical evolutions of two-soliton solutions (3.20) and (3.21) at times $t=-3, t=0$ and $t=3$. Figures $4-6$ show that two-soliton solutions (3.20) and (3.21) possess time-varying amplitudes in the process of propagations. 

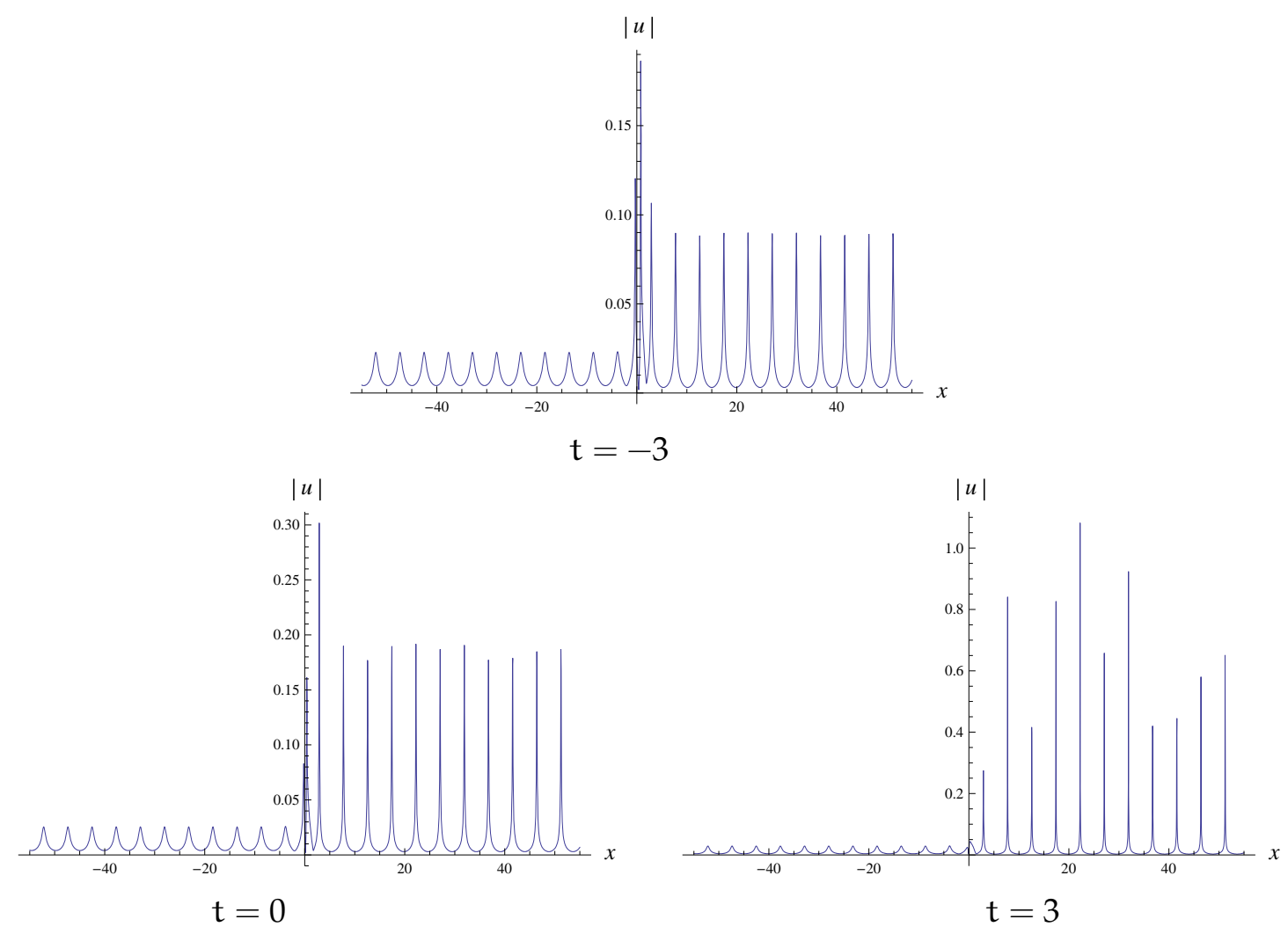

Figure 5: Dynamical evolutions of two-soliton solution (3.20).
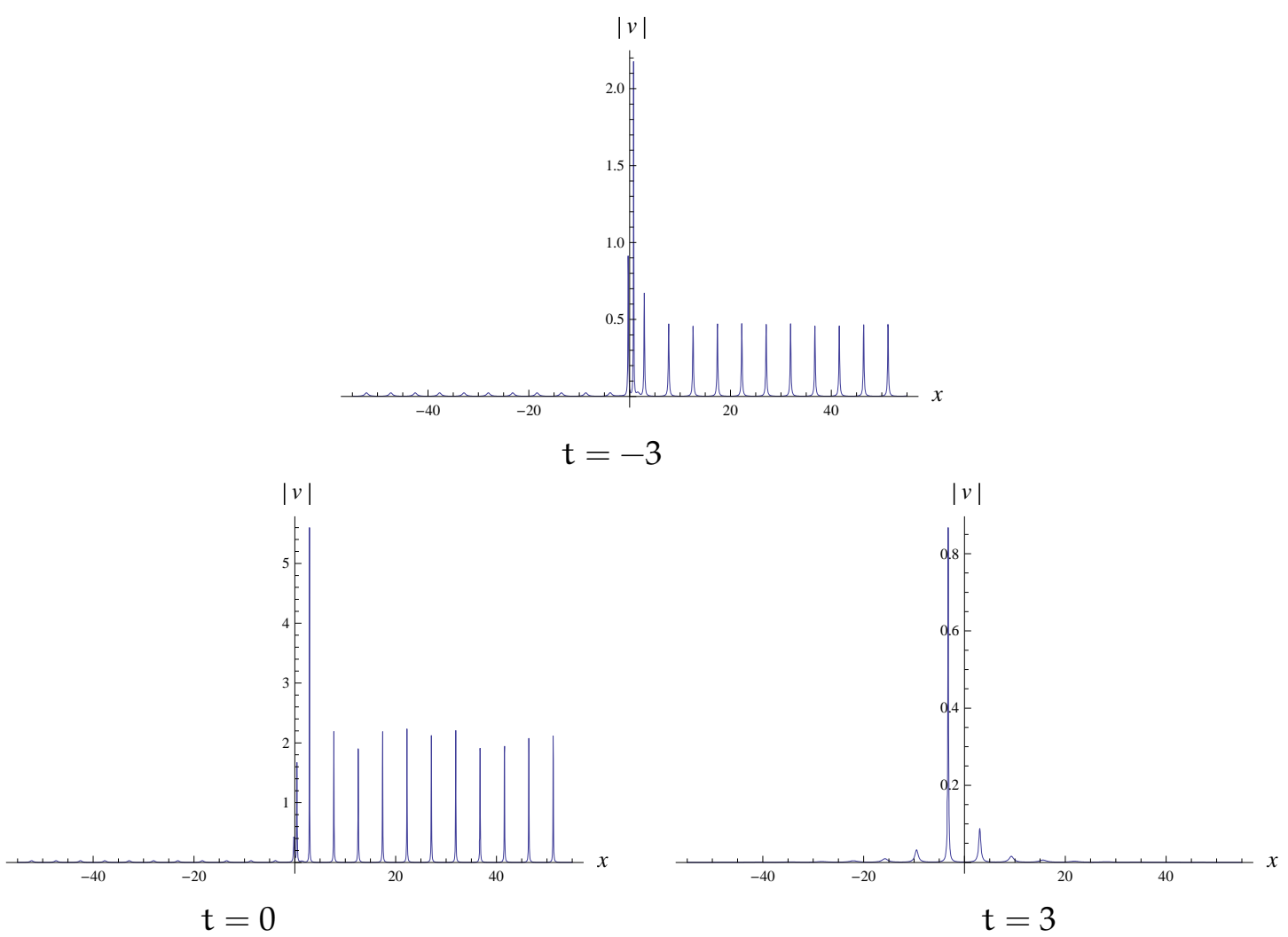

Figure 6: Dynamical evolutions of two-soliton solution (3.21). 
Similarly, we can determine three-soliton solutions of (1.1) and (1.2) as follows:

$$
\begin{gathered}
u=a_{0} \frac{g_{3 x} f_{3}-f_{3 x} g_{3}}{f_{3} g_{3}}, \\
v=-a_{0}^{2} \frac{\gamma_{1}(t)}{\gamma_{2}(t)} \frac{g_{3} h_{3}}{f_{3}^{2}}+a_{0} \frac{a_{0} \gamma_{1}(t)-2 \gamma_{3}(t)}{2 \gamma_{2}(t)}\left(\frac{f_{3 x}^{2}}{f_{3}^{2}}-\frac{g_{3 x}^{2}}{g_{3}^{2}}-\frac{f_{3 x x}}{f_{3}}+\frac{g_{3 x x}}{g_{3}}\right),
\end{gathered}
$$

by

$$
\begin{aligned}
& f_{3}=1+e^{\xi_{1}+\eta_{1}+\theta_{14}}+e^{\xi_{1}+\eta_{2}+\theta_{15}}+e^{\xi_{1}+\eta_{3}+\theta_{16}}+e^{\xi_{2}+\eta_{1}+\theta_{24}}+e^{\xi_{2}+\eta_{2}+\theta_{25}} \\
& +e^{\xi_{2}+\eta_{3}+\theta_{26}}+e^{\xi_{3}+\eta_{1}+\theta_{34}}+e^{\xi_{3}+\eta_{2}+\theta_{35}}+e^{\xi_{3}+\eta_{3}+\theta_{36}} \\
& +e^{\xi_{1}+\xi_{2}+\eta_{1}+\eta_{2}+\theta_{12}+\theta_{14}+\theta_{15}+\theta_{24}+\theta_{25}+\theta_{45}}+e^{\xi_{1}+\xi_{3}+\eta_{1}+\eta_{3}+\theta_{13}+\theta_{14}+\theta_{16}+\theta_{34}+\theta_{36}+\theta_{46}} \\
& +e^{\xi_{1}+\xi_{3}+\eta_{2}+\eta_{3}+\theta_{13}+\theta_{15}+\theta_{16}+\theta_{35}+\theta_{36}+\theta_{56}}+e^{\xi_{2}+\xi_{3}+\eta_{1}+\eta_{2}+\theta_{23}+\theta_{24}+\theta_{25}+\theta_{34}+\theta_{35}+\theta_{45}} \\
& +e^{\xi_{2}+\xi_{3}+n_{1}+\eta_{3}+\theta_{23}+\theta_{24}+\theta_{26}+\theta_{34}+\theta_{36}+\theta_{46}}+e^{\xi_{2}+\xi_{3}+n_{2}+\eta_{3}+\theta_{23}+\theta_{25}+\theta_{26}+\theta_{35}+\theta_{36}+\theta_{56}} \\
& +e^{\xi_{1}+\xi_{2}+\xi_{3}+\eta_{1}+\eta_{2}+\eta_{3}+\theta_{12}+\theta_{13}+\theta_{14}+\theta_{15}+\theta_{16}+\theta_{23}+\theta_{24}+\theta_{25}+\theta_{26}+\theta_{34}+\theta_{35}+\theta_{36}+\theta_{45}+\theta_{46}+\theta_{56},} \\
& g_{3}=e^{\xi_{1}}+e^{\xi_{2}}+e^{\xi_{3}}+e^{\xi_{1}+\xi_{2}+\xi_{3}+\eta_{1}+\eta_{2}+\theta_{12}+\theta_{13}+\theta_{14}+\theta_{15}+\theta_{23}+\theta_{24}+\theta_{25}+\theta_{34}+\theta_{35}+\theta_{45}} \\
& +e^{\xi_{1}+\xi_{2}+\xi_{3}+\eta_{1}+n_{3}+\theta_{12}+\theta_{13}+\theta_{14}+\theta_{16}+\theta_{23}+\theta_{24}+\theta_{26}+\theta_{34}+\theta_{36}+\theta_{46}} \\
& +e^{\xi_{1}+\xi_{2}+\xi_{3}+\eta_{2}+\eta_{3}+\theta_{12}+\theta_{13}+\theta_{15}+\theta_{16}+\theta_{23}+\theta_{25}+\theta_{26}+\theta_{35}+\theta_{36}+\theta_{56}} \\
& +e^{\xi_{1}+\xi_{2}+\eta_{1}+\theta_{12}+\theta_{14}+\theta_{24}}+e^{\xi_{1}+\xi_{2}+\eta_{2}+\theta_{12}+\theta_{15}+\theta_{25}}+e^{\xi_{1}+\xi_{2}+\eta_{3}+\theta_{12}+\theta_{16}+\theta_{26}} \\
& +e^{\xi_{1}+\xi_{3}+\eta_{1}+\theta_{13}+\theta_{14}+\theta_{34}}+e^{\xi_{1}+\xi_{3}+\eta_{2}+\theta_{13}+\theta_{15}+\theta_{35}}+e^{\xi_{1}+\xi_{3}+\eta_{3}+\theta_{13}+\theta_{16}+\theta_{36}} \\
& +e^{\xi_{2}+\xi_{3}+\eta_{1}+\theta_{23}+\theta_{24}+\theta_{34}}+e^{\xi_{2}+\xi_{3}+\eta_{2}+\theta_{23}+\theta_{25}+\theta_{35}}+e^{\xi_{2}+\xi_{3}+\eta_{3}+\theta_{23}+\theta_{26}+\theta_{36}}, \\
& h_{3}=e^{\eta_{1}}+e^{\eta_{2}}+e^{\eta_{3}}+e^{\xi_{1}+\xi_{2}+\eta_{1}+\eta_{2}+\eta_{3}+\theta_{12}+\theta_{14}+\theta_{15}+\theta_{16}+\theta_{24}+\theta_{25}+\theta_{26}+\theta_{45}+\theta_{46}+\theta_{56}} \\
& +e^{\xi_{1}+\xi_{3}+\eta_{1}+\eta_{2}+\eta_{3}+\theta_{13}+\theta_{14}+\theta_{15}+\theta_{16}+\theta_{34}+\theta_{35}+\theta_{36}+\theta_{45}+\theta_{46}+\theta_{56}} \\
& +e^{\xi_{2}+\xi_{3}+\eta_{1}+\eta_{2}+\eta_{3}+\theta_{23}+\theta_{24}+\theta_{25}+\theta_{26}+\theta_{34}+\theta_{35}+\theta_{36}+\theta_{45}+\theta_{46}+\theta_{56}} \\
& +e^{\xi_{1}+\eta_{1}+\eta_{2}+\theta_{14}+\theta_{15}+\theta_{45}}+e^{\xi_{2}+\eta_{1}+\eta_{2}+\theta_{24}+\theta_{25}+\theta_{45}}+e^{\xi_{3}+\eta_{1}+\eta_{2}+\theta_{34}+\theta_{35}+\theta_{45}} \\
& +e^{\xi_{1}+\eta_{1}+\eta_{3}+\theta_{14}+\theta_{16}+\theta_{46}}+e^{\xi_{2}+\eta_{1}+\eta_{3}+\theta_{24}+\theta_{26}+\theta_{46}}+e^{\xi_{3}+\eta_{1}+\eta_{3}+\theta_{34}+\theta_{36}+\theta_{46}} \\
& +e^{\xi_{1}+\eta_{2}+\eta_{3}+\theta_{15}+\theta_{16}+\theta_{56}}+e^{\xi_{2}+\eta_{2}+\eta_{3}+\theta_{25}+\theta_{26}+\theta_{56}}+e^{\xi_{3}+\eta_{2}+\eta_{3}+\theta_{35}+\theta_{36}+\theta_{56}},
\end{aligned}
$$

where

$$
\begin{gathered}
\xi_{i}=k_{i} x-\frac{1}{2} a_{0} k_{i}^{2} \int \gamma_{1}(t) d t, \quad \eta_{i}=l_{i} x+\frac{1}{2} a_{0} l_{i}^{2} \int \gamma_{1}(t) d t, \quad(i=1,2,3), \\
e^{\theta_{i j}}=-\left(k_{i}-k_{j}\right)^{2}, \quad(i<j=2,3), \\
e^{\theta_{i(j+n)}}=-\frac{1}{\left(k_{i}+l_{j}\right)^{2}}, \quad(i, j=1,2,3), \\
e^{\theta_{(i+n)(j+n)}}=-\left(l_{i}-l_{j}\right)^{2}, \quad(i<j=2,3) .
\end{gathered}
$$

Selecting $k_{1}=i, k_{2}=0.3 i, k_{3}=2 i, l_{1}=2, l_{2}=1, l_{3}=-1.5, a_{0}=0.005, \gamma_{1}(t)=0.5 e^{0.5 t^{2}}, \gamma_{2}(t)=2 e^{0.5 t^{2}}$ and $\gamma_{3}(t)=e^{0.5 t^{2}}$, we show in Figure 7 the spatial structures of three-soliton solutions (3.22) and (3.23). In Figures 8 and 9, the corresponding dynamical evolutions of three-soliton solutions (3.22) and (3.23) are described at times $t=-3, t=0$ and $t=3$. From Figures 7-9 we can see that three-soliton solutions (3.22) 
and (3.23) possess time-varying amplitudes in the process of propagations.
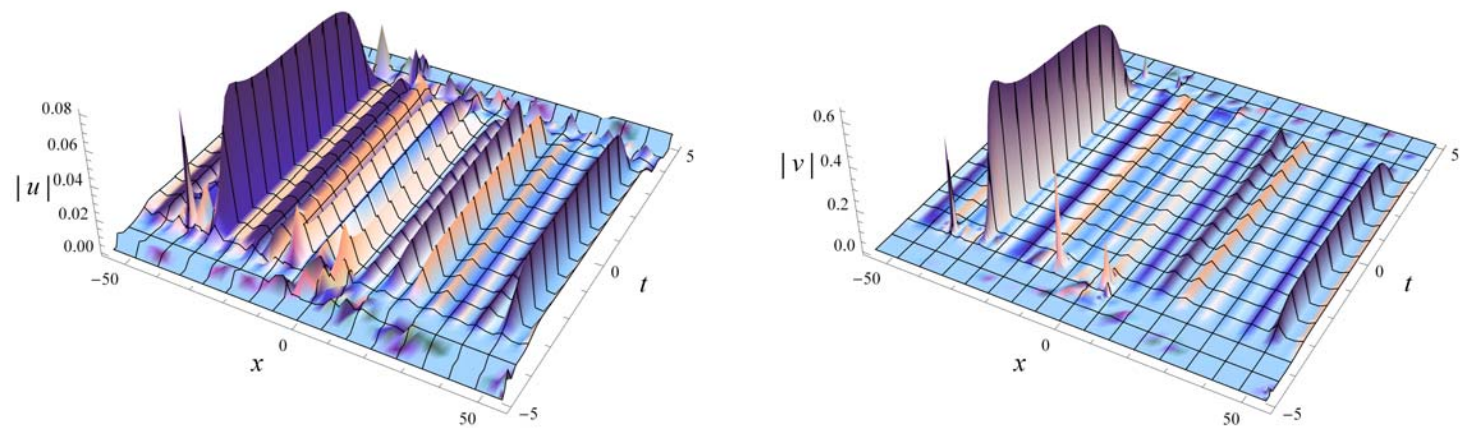

Figure 7: Spatial structures of bright and dark three-soliton solutions (3.22) and (3.23).
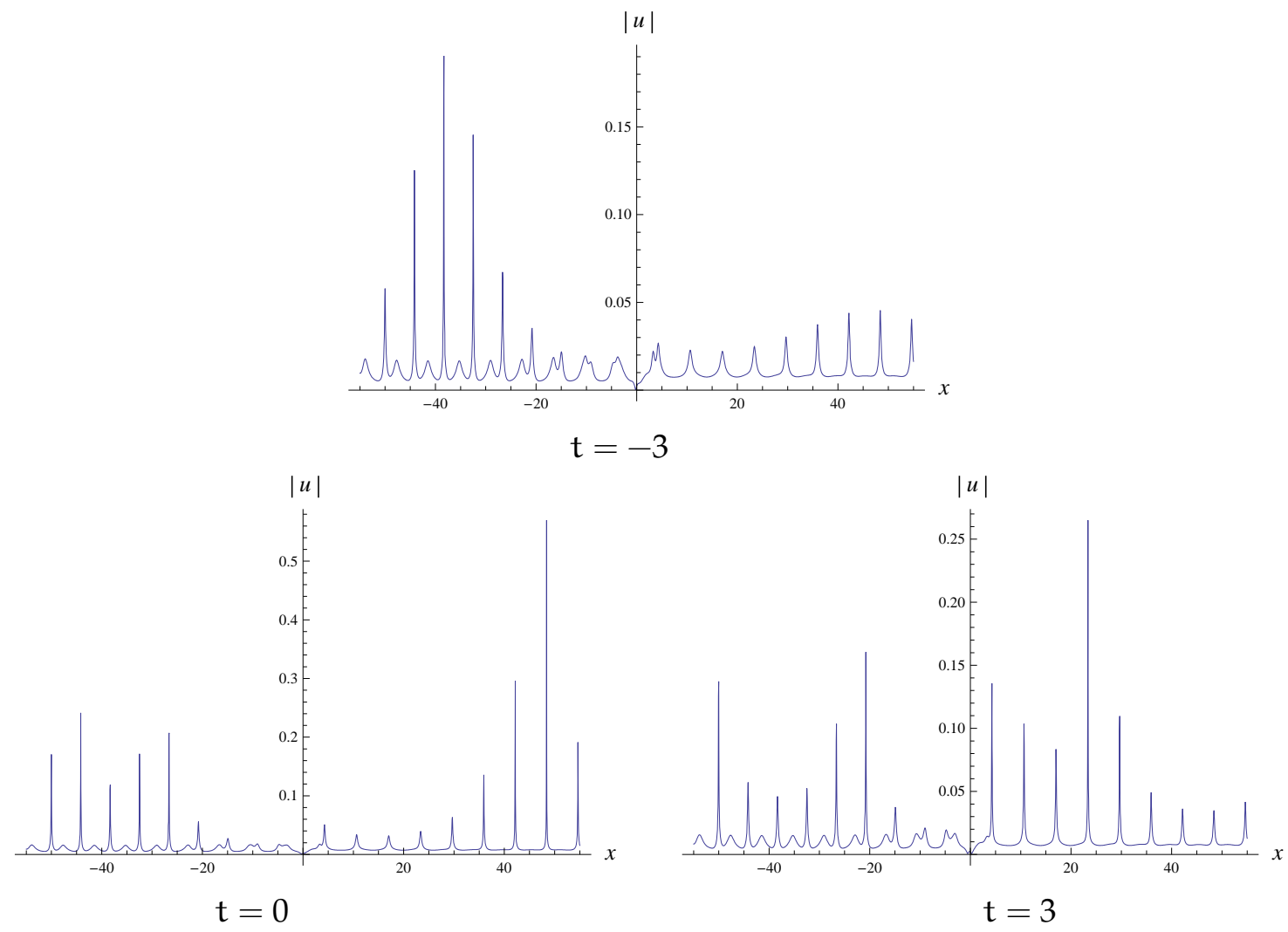

Figure 8: Dynamical evolutions of three-soliton solution (3.22).

Generally speaking, if we take

$$
\begin{aligned}
& g^{(1)}=e^{\xi_{1}}+e^{\xi_{2}}+\cdots+e^{\xi_{n}}, \quad \xi_{i}=k_{i}-\frac{1}{2} a_{0} k_{i}^{2} \int \gamma_{1}(t) d t, \\
& h^{(1)}=e^{\eta_{1}}+e^{\eta_{2}}+\cdots+e^{\eta_{n}}, \quad \eta_{i}=l_{i}+\frac{1}{2} a_{0} l_{i}^{2} \int \gamma_{1}(t) d t, \\
& f_{n}=\sum_{\mu=0,1} Z_{1}(\mu) e^{\sum_{i=1}^{2 n} \mu_{i} \xi_{i}}+\sum_{1 \leqslant i<j}^{2 n} \mu_{i} \mu_{j} \theta_{i j},
\end{aligned}
$$



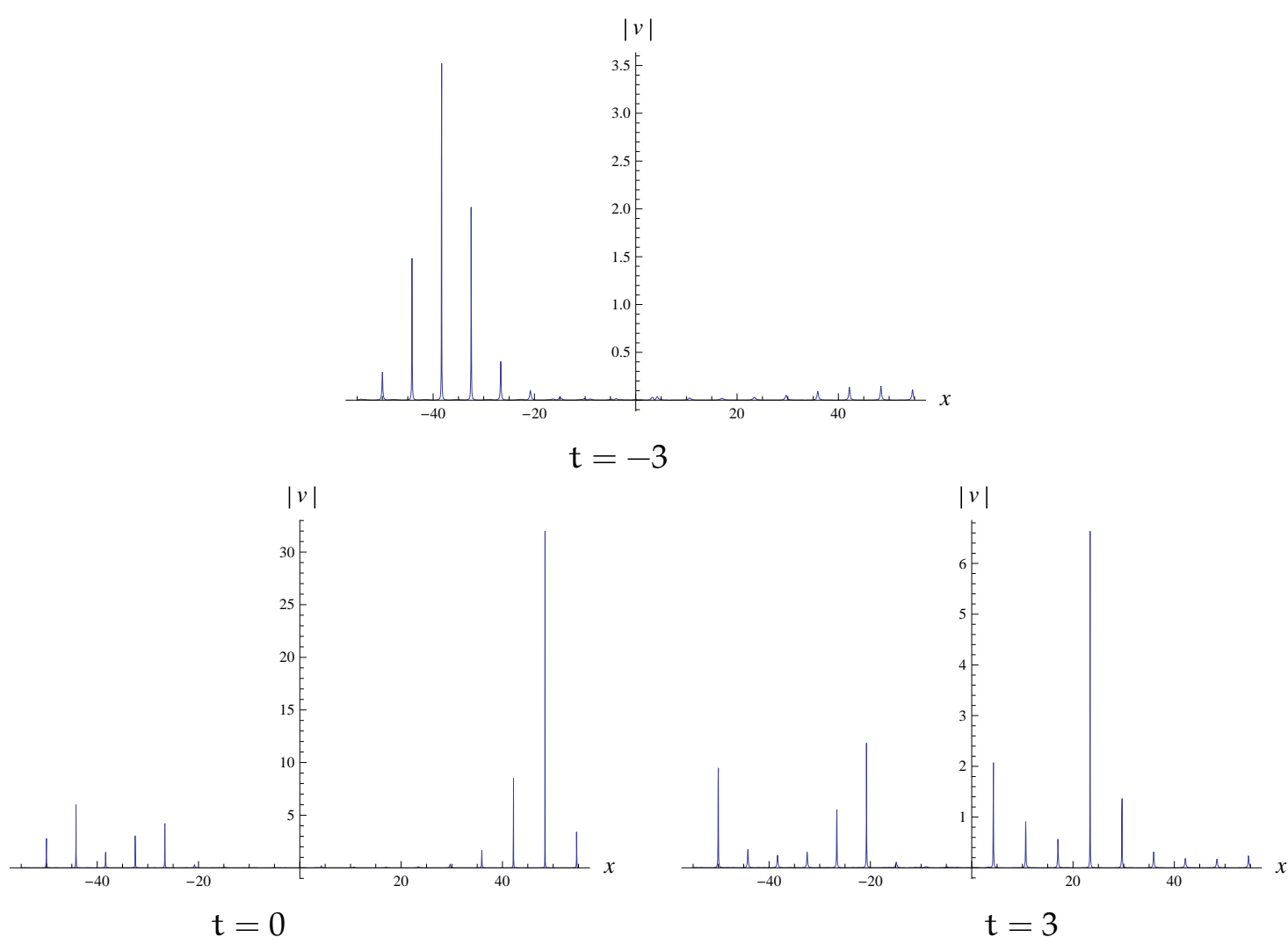

Figure 9: Dynamical evolutions of three-soliton solution (3.23).

$$
\begin{aligned}
& g_{n}=\sum_{\mu=0,1} Z_{2}(\mu) e^{\sum_{i=1}^{2 n} \mu_{i} \xi_{i}}+\sum_{1 \leqslant i<j}^{2 n} \mu_{i} \mu_{j} \theta_{i j}, \\
& h_{n}=\sum_{\mu=0,1} Z_{3}(\mu) e^{\sum_{i=1}^{2 n} \mu_{i} \xi_{i}}+\sum_{1 \leqslant i<j}^{2 n} \mu_{i} \mu_{j} \theta_{i j}, \\
& \xi_{i}=k_{i} x-\frac{1}{2} a_{0} k_{i}^{2} \int \gamma_{1}(t) d t, \quad \eta_{i}=l_{i} x+\frac{1}{2} a_{0} l_{i}^{2} \int \gamma_{1}(t) d t, \quad(i=1,2, \cdots, n), \\
& \xi_{n+j}=\eta_{j}, \quad(j=1,2, \cdots, n), \\
& e^{\theta_{i(j+n)}}=-\frac{1}{\left(k_{i}+l_{j}\right)^{2}}, \quad(i, j=1,2, \cdots, n), \\
& e^{\theta_{i j}}=-\left(k_{i}-k_{j}\right)^{2}, \quad e^{\theta_{(i+n)(j+n)}}=-\left(l_{i}-l_{j}\right)^{2}, \quad(i<j=2,3, \cdots, n),
\end{aligned}
$$

we can give uniform formulae of $n$-soliton solutions of (1.1) and (1.2) as follows:

$$
\begin{gathered}
u=a_{0} \frac{g_{n x} f_{n}-f_{n x} g_{n}}{f_{n} g_{n}}, \\
v=-a_{0}^{2} \frac{\gamma_{1}(t)}{\gamma_{2}(t)} \frac{g_{n} h_{n}}{f_{n}^{2}}+a_{0} \frac{a_{0} \gamma_{1}(t)-2 \gamma_{3}(t)}{2 \gamma_{2}(t)}\left(\frac{f_{n x}^{2}}{f_{n}^{2}}-\frac{g_{n x}^{2}}{g_{n}^{2}}-\frac{f_{n x x}}{f_{n}}+\frac{g_{n x x}}{g_{n}}\right),
\end{gathered}
$$

where the summation $\sum_{\mu=0,1}$ refers to all possible combinations of each $\mu_{i}=0,1$ for $i=1,2, \cdots, n$, and $Z_{1}(\mu), Z_{2}(\mu)$ and $Z_{3}(\mu)$ denote that when we select all the possible combinations $\mu_{j}(j=1,2, \cdots, 2 n)$ the following conditions hold, respectively: 


$$
\sum_{j=1}^{n} \mu_{j}=\sum_{j=1}^{n} \mu_{n+j}, \quad \sum_{j=1}^{n} \mu_{j}=\sum_{j=1}^{n} \mu_{n+j}+1, \quad \sum_{j=1}^{n} \mu_{j}+1=\sum_{j=1}^{n} \mu_{n+j} .
$$

To the best of our knowledge, the obtained one-soliton solutions (3.18) and (3.19), two-soliton solutions (3.20) and (3.21), three-soliton solutions (3.22) and (3.23) and $n$-soliton solutions (3.24) and (3.25) are new, they have not been reported in literature.

\section{Conclusions}

Based on (3.2) and (3.3), a special case of the bilinear forms (2.9) and (2.10) under the condition (3.1), we have successfully extended Hirota's bilinear method to the new time-dependent-coefficient WBK (1.1) and (1.2). As a result, new one-soliton solutions (3.18) and (3.19), two-soliton solutions (3.20) and (3.21), three-soliton solutions (3.22) and (3.23) and the uniform formulae of $n$-soliton solutions (3.24) and (3.25) are obtained. For the existing solutions in $[2,7-9,11,24,25,27,30,34-36,40,41,48,49,51-53,57,70]$, some of them can be recovered as special cases of the results obtained in the present study. Here we take the following solutions [48]

$$
\begin{gathered}
u=-k \sqrt{\alpha+\beta^{2}} \operatorname{coth} k(x-\lambda t+c)+\lambda, \\
v=-2 k^{2} \sqrt{\alpha+\beta^{2}}\left(\beta+\sqrt{\alpha+\beta^{2}}\right) \operatorname{csch}^{2} k(x-\lambda t+c)+\lambda,
\end{gathered}
$$

as the first example. In this case, we select $\gamma_{1}(t)=1, \gamma_{2}(t)=1, \gamma_{3}(t)=\beta, a_{0}=2 \sqrt{\alpha+\beta^{2}}, k_{1}=$ $k+\lambda /\left(2 \sqrt{\alpha+\beta^{2}}\right), l_{1}=k-\lambda /\left(2 \sqrt{\alpha+\beta^{2}}\right)$ and set the integration constants of (3.16) and (3.17) as $\ln 4 k^{2} / \lambda$, then a direct computation shows that the one-soliton solutions (3.18) and (3.19) arrive at solutions (4.1) and (4.2). In the second example, we select $\gamma_{1}(t)=1, \gamma_{2}(t)=1, \gamma_{3}(t)=\beta, a_{0}=-2 \sqrt{\alpha+\beta^{2}}, k_{1}=4$, $l_{1}=-2$ and set the integration constants of (3.16) and (3.17) as $-\theta_{13} / 6 \sqrt{2}$, then the one-soliton solutions (3.18) and (3.19) give the known solutions [27]

$$
\begin{aligned}
& u=2 \sqrt{2}[\tanh (x+6 \sqrt{2} t)-3] \\
& v=-8(\sqrt{2}-2) \operatorname{sech}^{2}(x+6 \sqrt{2} t) .
\end{aligned}
$$

The third example is based on (2.1), (2.2), (2.3), (2.4), (2.5), (2.6), (2.11), (3.2) and (3.3). Setting $\gamma_{1}(t)=1$, $\gamma_{2}(t)=1, \gamma_{3}(t)=\beta, a_{0}=-2 \sqrt{\alpha+\beta^{2}}$, from (2.1) and (2.2) we then have

$$
\begin{gathered}
u=-2 \sqrt{\alpha+\beta^{2}}(\ln A)_{x,} \\
v=-4\left(\alpha+\beta^{2}\right) A B+2\left[\alpha+\beta\left(\beta+\sqrt{\alpha+\beta^{2}}\right)\right](\ln A)_{x x},
\end{gathered}
$$

where $A$ and $B$ satisfy the constant-coefficient AKNS equations

$$
\begin{aligned}
& A_{t}=-\sqrt{\alpha+\beta^{2}}\left(2 A^{2} B-A_{x x}\right), \\
& B_{t}=-\sqrt{\alpha+\beta^{2}}\left(-2 A B^{2}+B_{x x}\right) .
\end{aligned}
$$

In view of (2.11) and (3.1), we reduce (3.2) and (3.3) as

$$
\begin{aligned}
& D_{t} g \cdot f=-\sqrt{\alpha+\beta^{2}} D_{x}^{2} g \cdot f, \\
& D_{t} h \cdot f=\sqrt{\alpha+\beta^{2}} D_{x}^{2} h \cdot f .
\end{aligned}
$$

With the help of (2.11), (4.3), (4.4), (4.5), (4.6), (4.7), (4.8), we can obtain the following multi-soliton solutions [27] expressed by double Wronskian determinants

$$
u=-2 \sqrt{\alpha+\beta^{2}}\left[\ln \left(2 \frac{|\widehat{N-2} ; \widehat{M}|}{|\widehat{N-1} ; \widehat{M-1}|}\right)\right]_{x},
$$




$$
v=16\left(\alpha+\beta^{2}\right) \frac{|\widehat{N-2} ; \widehat{M}|}{|\widehat{N-1} ; \widehat{M-1}|} \frac{|\widehat{N-2} ; \widehat{M}|}{|\widehat{N-1} ; \widehat{M-1}|}+2\left[\alpha+\beta\left(\beta+\sqrt{\alpha+\beta^{2}}\right)\right]\left[\ln \left(2 \frac{|\widehat{N-2} ; \widehat{M}|}{|\widehat{N-1} ; \widehat{M-1}|}\right)\right]_{x x},
$$

under the linear Wronskian conditions

$$
\begin{gathered}
\varphi_{j x}=-k_{j} \varphi_{j}, \quad \varphi_{j t}=-2 \sqrt{\alpha+\beta^{2}} \varphi_{j x x} \\
\psi_{j x}=k_{j} \psi_{j}, \quad \psi_{j t}=2 \sqrt{\alpha+\beta^{2}} \psi_{j x x}, \quad(j=1,2, \cdots, N+M+2),
\end{gathered}
$$

where the double Wronskian determinant is defined as

$$
W^{N, M}(\varphi ; \psi)=\operatorname{det}\left(\varphi, \partial \varphi, \cdots, \partial^{N-1} \varphi ; \psi, \partial \psi, \cdots, \partial^{M-1} \psi\right)=|\widehat{N-1} ; \widehat{M-1}|,
$$

with $\varphi=\left(\varphi_{1}, \varphi_{2}, \cdots, \varphi_{\mathrm{N}+M+2}\right)^{\mathrm{T}}$ and $\psi=\left(\psi_{1}, \psi_{2}, \cdots, \psi_{\mathrm{N}+M+2}\right)^{\mathrm{T}}$.

In the procedure of extending Hirota's bilinear method to (1.1) and (1.2), one of the key steps is to reduce (1.1) and (1.2) to the bilinear forms (2.9) and (2.10) by the transformations (2.7), (2.8) and (2.11). It is graphically shown that the dynamical evolutions of one-soliton solutions (3.18) and (3.19), two-soliton solutions (3.20) and (3.21), three-soliton solutions (3.22) and (3.23) possess time-varying amplitudes as Serkin et al. [37-39] reported in the process of propagations. Recently, fractional-order differential calculus and its applications have attached much attention [3, 26, 54, 71]. How to construct multi-soliton solutions of nonlinear PDEs with fractional derivatives through Hirota's bilinear method is worthy of study.

\section{Acknowledgment}

This work was supported by the Natural Science Foundation of China (11547005), the PhD Start-up Fund of Liaoning Province of China (20141137), the Liaoning BaiQianWan Talents Program of Liaoning Province of China (2013921055) and the Natural Science Foundation of Liaoning Province of China (20170540007).

\section{References}

[1] M. J. Ablowitz, P. A. Clarkson, Solitons, nonlinear evolution equations and inverse scattering, London Mathematical Society Lecture Note Series, Cambridge University Press, Cambridge, (1991). 1

[2] M. Arshad, A. R. Seadawy, D.-C. Lu, J. Wang, Travelling wave solutions of Drinfeld-Sokolov-Wilson, Whitham-BroerKaup and (2+1)-dimensional Broer-Kaup-Kupershmit equations and their applications, Chin. J. Phys., (2017), (In press). 1,4

[3] D. Baleanu, B. Agheli, R. Darzi, Analysis of the new technique to solution of fractional wave-and heat-like equation, Acta Phys. Polon. B, 48 (2017), 77-95. 4

[4] D. Baleanu, B. Kilic, M. Inc, The first integral method for Wu-Zhang nonlinear system with time-dependent coefficients, Proc. Rom. Acad. Ser. A Math. Phys. Tech. Sci. Inf. Sci., 16 (2015), 160-167. 1

[5] D. Y. Chen, Introduction of soliton, (Chinese), Science Press, Beijing, (2006). 1

[6] S.-H. Chen, P. Grelu, D. Mihalache, F. Baronio, Families of rational solutions of the Kadomtsev-Petviashvili equation, Romanian Rep. Phys., 68 (2016), 1407-1424. 1

[7] Y. Chen, Q. Wang, Multiple Riccati equations rational expansion method and complexiton solutions of the Whitham-BroerKaup equation, Phys. Lett. A, 347 (2005), 215-227. 1, 4

[8] Y. Chen, Q. Wang, B. Li, A generalized method and general form solutions to the Whitham-Broer-Kaup equation, Chaos Solitons Fractals, 22 (2004), 675-682. 1

[9] Y. Chen, Q. Wang, B. Li, Elliptic equation rational expansion method and new exact travelling solutions for WhithamBroer-Kaup equations, Chaos Solitons Fractals, 26 (2005), 231-246. 1, 4

[10] D. Y. Chen, X. Y. Zhu, J. B. Zhang, Y. Y. Sun, Y. Shi, New soliton solutions to isospectral AKNS equations, (Chinese) ; translated from Chinese Ann. Math. Ser. A, 33 (2012), 205-216, Chinese J. Contemp. Math., 33 (2012), 167-176. 1

[11] S. M. El-Sayed, D. Kaya, Exact and numerical traveling wave solutions of Whitham-Broer-Kaup equations, Appl. Math. Comput., 167 (2005), 1339-1349. 1, 4

[12] E.-G. Fan, Travelling wave solutions in terms of special functions for nonlinear coupled evolution systems, Phys. Lett. A, 300 (2002), 243-249. 1 
[13] C. S. Gardner, J. M. Greene, M. D. Kruskal, R. M. Miura, Method for solving the Korteweg-deVries equation, Phys. Rev. Lett., 19 (1967), 1095-1097. 1

[14] J.-H. He, X.-H. Wu, Exp-function method for nonlinear wave equations, Chaos Solitons Fractals, 30 (2006), 700-708. 1

[15] R. Hirota, Exact solution of the Korteweg-de Vries equation for multiple collisions of solitons, Phys. Rev. Lett., 27 (1971), 1192-1194. 1

[16] R. Hirota, Exact solution of the modified Korteweg-de Vries equation for multiple collisions of solitons, J. Phys. Soc. Japan, 33 (1972), 1456-1458. 1

[17] M. Inc, Constructing solitary pattern solutions of the nonlinear dispersive Zakharov-Kuznetsov equation, Chaos Solitons Fractals, 39 (2009), 109-119. 1

[18] M. Inç, On new exact special solutions of the GNLS(m, n, p, q) equations, Modern Phys. Lett. B, 24 (2010), $1769-1783$.

[19] M. Inc, Compact and noncompact structures of a three-dimensional $3 \mathrm{DKP}(\mathrm{m}, \mathrm{n})$ equation with nonlinear dispersion, Appl. Math. Lett., 26 (2013), 437-444.

[20] M. Inc, Some special structures for the generalized nonlinear Schrödinger equation with nonlinear dispersion, Waves Random Complex Media, 23 (2013), 77-88.

[21] M. Inc, E. Ates, Optical soliton solutions for generalized NLSE by using Jacobi elliptic functions, Optoelectron. Adv. Mat., 9 (2015), 1081-1087. 1

[22] M. Inc, B. Kilic, D. Baleanu, Optical soliton solutions of the pulse propagation generalized equation in parabolic-law media with space-modulated coefficients, Optik, 127 (2016), 1056-1058. 1

[23] M. Inc, Z. S. Korpinar, M. M. Al Qurashi, D. Baleanu, A new method for approximate solutions of some nonlinear equations: residual power series method, Adv. Mech. Eng., 8 (2016), 8 pages. 1

[24] X.-Y. Jiao, H.-Q. Zhang, An extended method and its application to Whitham-Broer-Kaup equation and two-dimensional perturbed KdV equation, Appl. Math. Comput., 172 (2006), 664-677. 1, 4

[25] M. Khalfallah, Exact traveling wave solutions of the Boussinesq-Burgers equation, Math. Comput. Modelling, 49 (2009), 666-671. 1, 1, 4

[26] D. Kumar, J. Singh, D. Baleanu, A hybrid computational approach for Klein-Gordon equations on Cantor sets, Nonlinear Dynam., 87 (2017), 511-517. 4

[27] G.-D. Lin, Y.-T. Gao, L. Wang, D.-X. Meng, X. Yu, Elastic-inelastic-interaction coexistence and double Wronskian solutions for the Whitham-Broer-Kaup shallow-water-wave model, Commun. Nonlinear Sci. Numer. Simul., 16 (2011), 3090-3096. 1, 4, 4, 4

[28] Y.-B. Liu, A. S. Fokas, D. Mihalache, J.-S. He, Parallel line rogue waves of the third-type Davey-Stewartson equation, Romanian Rep. Phys., 68 (2016), 1425-1446. 1

[29] Q. P. Liu, X.-B. Hu, M.-X. Zhang, Supersymmetric modified Korteweg-de Vries equation: bilinear approach, Nonlinearity, 18 (2005), 1597-1603. 1

[30] Y. Liu, X.-Q. Liu, Exact solutions of Whitham-Broer-Kaup equations with variable coefficients, Acta Phys. Sin., 63 (2014), 9 pages. $1,1,1,4$

[31] V. B. Matveev, M. A. Salle, Darboux transformations and solitons, Springer Series in Nonlinear Dynamics, SpringerVerlag, Berlin, (1991). 1

[32] I. N. McArthur, C. M. Yung, Hirota bilinear form for the super-KdV hierarchy, Modern Phys. Lett. A, 8 (1993), 17391745. 1

[33] M. R. Miura, Bäcklund transformation, Springer-Verlag, Berlin, (1978). 1

[34] A. Mohebbi, Z. Asgari, M. Dehghan, Numerical solution of nonlinear Jaulent-Miodek and Whitham-Broer-Kaup equations, Commun. Nonlinear Sci. Numer. Simul., 17 (2012), 4602-4610. 1, 4

[35] S. T. Mohyud-Din, A. Yildırım, G. Demirli, Traveling wave solutions of Whitham-Broer-Kaup equations by homotopy perturbation method, J. King Saud Univ. Sci., 22 (2010), 173-176. 1

[36] M. Rafei, H. Daniali, Application of the variational iteration method to the Whitham-Broer-Kaup equations, Comput. Math. Appl., 54 (2007), 1079-1085. 1, 4

[37] V. N. Serkin, A. Hasegawa, Novel soliton solutions of the nonlinear Schrödinger equation model, Phys. Rev. Lett., 85 (2000), 4502-4505. 4

[38] V. N. Serkin, A. Hasegawa, T. L. Belyaeva, Nonautonomous solitons in external potentials, Phys. Rev. Lett., 98 (2007), 4 pages.

[39] V. N. Serkin, A. Hasegawa, T. L. Belyaeva, Nonautonomous matter-wave solitons near the Feshbach resonance, Phys. Rev. A, 81 (2010), 19 pages. 4

[40] J.-W. Shen, W. Xu, Y.-F. Jin, Bifurcation method and traveling wave solution to Whitham-Broer-Kaup equation, Appl. Math. Comput., 171 (2005), 677-702. 1, 4

[41] M. Song, J. Cao, X.-L. Guan, Application of the bifurcation method to the Whitham-Broer-Kaup-like equations, Math. Comput. Modelling, 52 (2012), 688-696. 1, 4

[42] H. Triki, H. Leblond, D. Mihalache, Soliton solutions of nonlinear diffusion-reaction-type equations with time-dependent coefficients accounting for long-range diffusion, Nonlinear Dynam., 86 (2016), 2115-2126. 1

[43] H. Triki, A.-M. Wazwaz, Soliton solutions of the cubic-quintic nonlinear Schrodinger equation with variable coefficients, Romanian J. Phys., 61 (2016), 360-366. 1

[44] M.-L. Wang, Exact solutions for a compound KdV-Burgers equation, Phys. Lett. A, 213 (1996), 279-287. 1

[45] A.-M. Wazwaz, The Hirota's bilinear method and the tanh-coth method for multiple-soliton solutions of the Sawada-Kotera- 
Kadomtsev-Petviashvili equation, Appl. Math. Comput., 200 (2008), 160-166. 1

[46] J. Weiss, M. Tabor, G. Carnevale, The Painlevé property for partial differential equations, J. Math. Phys., 24 (1983), 522-526. 1

[47] X.-Y. Wen, A new integrable lattice hierarchy associated with a discrete $3 \times 3$ matrix spectral problem: N-fold Darboux transformation and explicit solutions, Rep. Math. Phys., 71 (2013), 15-32. 1

[48] F.-D. Xie, Z.-Y. Yan, H.-Q. Zhang, Explicit and exact traveling wave solutions of Whitham-Broer-Kaup shallow water equations, Phys. Lett. A, 285 (2001), 76-80. 1, 4

[49] G.-Q. Xu, Z.-B. Li, Exact travelling wave solutions of the Whitham-Broer-Kaup and Broer-Kaup-Kupershmidt equations, Chaos Solitons Fractals, 24 (2005), 549-556. 1, 4

[50] S.-W. Xu, K. Porsezian, J.-S. He, Y. Cheng, Multi-optical rogue waves of the Maxwell-Bloch equations, Romanian Rep. Phys., 68 (2016), 316-340. 1

[51] Z.-L. Yan, X.-Q. Liu, Solitary wave and non-traveling wave solutions to two nonlinear evolution equations, Commun. Theor. Phys. (Beijing), 44 (2005), 479-482. 1, 1, 1, 4

[52] Z.-Y. Yan, H.-Q. Zhang, New explicit solitary wave solutions and periodic wave solutions for Whitham-Broer-Kaup equation in shallow water, Phys. Lett. A, 285 (2001), 355-362. 1, 1

[53] Z.-L. Yan, J.-P. Zhou, New explicit solutions of $(1+1)$-dimensional variable-coefficient Broer-Kaup system, Commun. Theor. Phys. (Beijing), 54 (2010), 965-970. 1, 1, 4

[54] X.-J. Yang, D. Baleanu, H. M. Srivastava, Local fractional integral transforms and their applications, Elsevier/Academic Press, Amsterdam, (2015). 4

[55] S. Zhang, Application of Exp-function method to a KdV equation with variable coefficients, Phys. Lett. A, 365 (2007), 448-453. 1

[56] S. Zhang, Exact solutions of a KdV equation with variable coefficients via Exp-function method, Nonlinear Dynam., 52 (2008), 11-17. 1

[57] P. Zhang, New exact solutions to breaking soliton equations and Whitham-Broer-Kaup equations, Appl. Math. Comput., 217 (2010), 1688-1696. 1, 4

[58] S. Zhang, B. Cai, Multi-soliton solutions of a variable-coefficient KdV hierarchy, Nonlinear Dynam., 78 (2014), 15931600. 1

[59] S. Zhang, M.-T. Chen, Painlevé integrability and new exact solutions of the $(4+1)$-dimensional Fokas equation, Math. Probl. Eng., 2015 (2015), 8 pages. 1

[60] S. Zhang, M.-T. Chen, W.-Y. Qian, Painlevé analysis for a forced Korteveg-de Vries equation arisen in fluid dynamics of internal solitary waves, Therm. Sci., 19 (2015), 1223-1226. 1

[61] S. Zhang, X.-D. Gao, Mixed spectral AKNS hierarchy from linear isospectral problem and its exact solutions, Open Phys., 13 (2015), 310-322. 1

[62] S. Zhang, X.-D. Gao, Exact N-soliton solutions and dynamics of a new AKNS equation with time-dependent coefficients, Nonlinear Dynam., 83 (2016), 1043-1052. 1

[63] S. Zhang, D. Liu, Multisoliton solutions of a $(2+1)$-dimensional variable-coefficient Toda lattice equation via Hirota's bilinear method, Canad. J. Phys., 92 (2014), 184-190. 1

[64] S. Zhang, D.-D. Liu, The third kind of Darboux transformation and multisoliton solutions for generalized Broer-Kaup equations, Turkish J. Phys., 39 (2015), 165-177. 1

[65] S. Zhang, C. Tian, W.-Y. Qian, Bilinearization and new multisoliton solutions for the $(4+1)$-dimensional Fokas equation, Pramana, 86 (2016), 1259-1267. 1

[66] S. Zhang, D. Wang, Variable-coefficient nonisospectral Toda lattice hierarchy and its exact solutions, Pramana, 85 (2015), 1143-1156. 1

[67] S. Zhang, T.-C. Xia, A generalized F-expansion method and new exact solutions of Konopelchenko-Dubrovsky equations, Appl. Math. Comput., 183 (2006), 1190-1200. 1

[68] S. Zhang, T.-C. Xia, A generalized auxiliary equation method and its application to $(2+1)$-dimensional asymmetric Nizhnik-Novikov-Vesselov equations, J. Phys. A, 40 (2007), 227-248. 1

[69] S. Zhang, B. Xu, H.-Q. Zhang, Exact solutions of a KdV equation hierarchy with variable coefficients, Int. J. Comput. Math., 91 (2014), 1601-1616. 1

[70] S. Zhang, H.-Q. Zhang, An Exp-function method for a new N-soliton solutions with arbitrary functions of a (2+1)dimensional vcBK system, Comput. Math. Appl., 61 (2011), 1923-1930. 1, 4

[71] S. Zhang, H.-Q. Zhang, Fractional sub-equation method and its applications to nonlinear fractional PDEs, Phys. Lett. A, 375 (2011), 1069-1073. 4

[72] S. Zhang, L.-Y. Zhang, Bilinearization and new multi-soliton solutions of $m K d V$ hierarchy with time-dependent coefficients, Open Phys., 14 (2016), 69-75. 1 